\title{
'It's not the destination, it's the journey that counts!'
}

Interview by Kate Quinlan

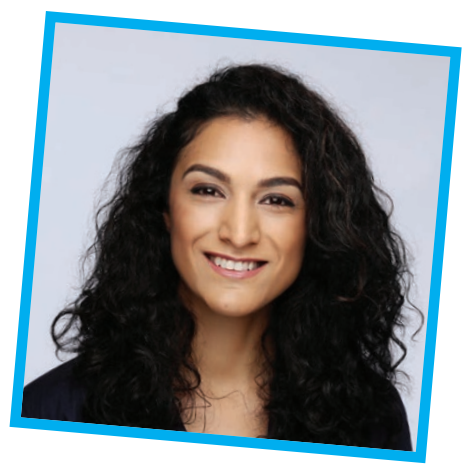

Dr Bella Dave is a Specialist Trainee Registrar in Oral Medicine at the Charles Clifford Dental Hospital and an Honorary Clinical Lecturer at the School of Clinical Dentistry in Sheffield. She is also a Collegiate Member of the Royal College of Physicians. She is the first Dental Trainee Representative appointed onto the Royal College of Surgeons of Edinburgh Dental Council.

$\mathrm{I}$ live in Sheffield, a 10-15 minute walk from work (it takes longer in the snow), but at present I do a one-day-a-week placement at Leeds Dental Institute, an hour's train journey away.

For breakfast I sometimes have toast or bagels, but always a banana and two cups of tea. When I am at Leeds I take my breakfast on the train; sometimes I take porridge - I'm sure all the other commuters love the smell of that!

Each week, every day is different. My week starts with a pain clinic on Monday morning, with a general oral medicine clinic in the afternoon. On the first Tuesday morning of the month there is a joint oral medicine/ paediatrics clinic. On the second Tuesday morning of the month I do either a joint oral medicine/dermatology clinic or a Behçet's clinic on a three monthly basis. The Behçet's clinic is a joint clinic with the dermatology and rheumatology teams to enable a more cohesive and streamlined approach to patient care. Its goal is that patients do not have to attend three different appointments with different teams for the same problem. For the rest of the month I have general oral medicine clinics on Tuesday mornings. On Tuesday afternoons I have administration time allocated which usually means that I am checking and signing off letters, reviewing results for patients seen in clinic or chasing results for patients on immunosuppressive systemic treatments which require monitoring in the community. This is a very time consuming job as we have to contact GPs and patients to find out when and if they have had their bloods taken, whether they have any further appointments and get the results faxed over to us. Sometimes I am also asked to see ward patients over at Royal Hallamshire Hospital - this can be very interesting as patients admitted to hospital often have more severe manifestations of disease. Making even a small improvement to their oral health to help them eat can result in a big difference to their recovery. Most of the patients I have seen were admitted under OMFS or haematology and I have also been asked to see patients in rheumatology clinics.

Even being able to differentiate whether a small lesion like a mouth ulcer is traumatic or due to medication (such as methotrexate for rheumatoid arthritis) does help guide the doctors in their management to ultimately of oro-mucosal disease. I have completed other attachments with the haematology and rheumatology departments and this also helps the physicians to understand what the speciality of oral medicine is and how we can help with their patients.

I initially trained as a doctor, with my core training in general internal medicine, as the intellectual challenge of medicine appealed to me more that the technical ones of surgery. I had wanted to be a dermatologist; however, in 2007 there were some major changes in junior doctor training as part of Modernising Medical

\section{'Making even a small improvement to their oral health to help them eat can result in a big difference to their recovery...'}

help the patient and this is really rewarding.

On Wednesdays I am at Leeds Dental

Institute to do more general clinics, but it is extremely valuable to have some variety in the units that I work in to broaden my experience of oral medicine. I am really lucky to gain training experience in a different oral medicine unit (there are not that many of them!). It is really important to gain a different perspective on how different clinicians practise so that you can develop your own way of practising.

Thursday and Friday mornings are again general oral medicine clinics. At the moment I spend Thursday afternoons doing a medical attachment with the gastroenterology department. This is because some gastro-intestinal conditions are often associated with oral manifestations of disease and it is also important for an oral medicine specialist to be able to differentiate between local and systemic causes
Careers. A rather flawed recruitment process meant that as a junior doctor, you were either in a run-through training placement or you were not and it was very much luck rather than merit which determined this. I was not very lucky and not having a run-through placement, I had very little chance of gaining a specialist training number in dermatology. So I did a significant amount of research around dermatology and other careers and I stumbled upon oral medicine, a speciality which sits at the interface of medicine and dentistry.

Oral medicine is very similar to dermatology, with opportunities for teaching and research. So I found out more about it and managed to attend some of Dr John Hamburger and Professor Joanna Zakrzewska's clinics. It sounded perfect - the only catch was that I would have to do a dental degree. At that time King's had recently launched their 
« three-year dental programme and oral medicine training as a registrar is three years. I was told that I could specialise straight after graduation so in the grand scheme of things, a total of six years training to become a consultant did not seem that long. Except that the three-year dental programme was oversubscribed that year so I managed to gain a place on the four-year course. So six years turned into seven. There was so much uncertainty in medical careers at the time, so suddenly many of the medical trainees felt it would be a good idea to diversify into dentistry or oral and maxillofacial surgery. This actually turned out to be a good thing though, as there were lots of other medics in my year of a similar age to me with a similar background who could understand my outlook. for an oral medicine training opportunity to appear, but having a broad scope of experience has helped me in so many different ways (I keep telling myself it's not the destination, it's the journey that counts!).

Dentists in secondary care face numerous challenges which can be very difficult to manage and quite different to those in general dental practice. This is especially true for trainees. If you are unhappy with a situation, you have three options: 1. Leave; 2. Do nothing; or 3. Do something.

In the past I have tried the first two options, so now I decided to try the third, which is why launched an online e-learning module which gives a good overview of the problem and how to tackle it. It can really help trainees to know that they are not alone and what steps they can take to improve the situation. This problem is more common in surgery but it also happens in dentistry and our dental trainee members should be aware of what support the College can offer. I myself have done the module and it really did make me think about the sort of clinician I want to be (if I ever finally do get a consultant job!).

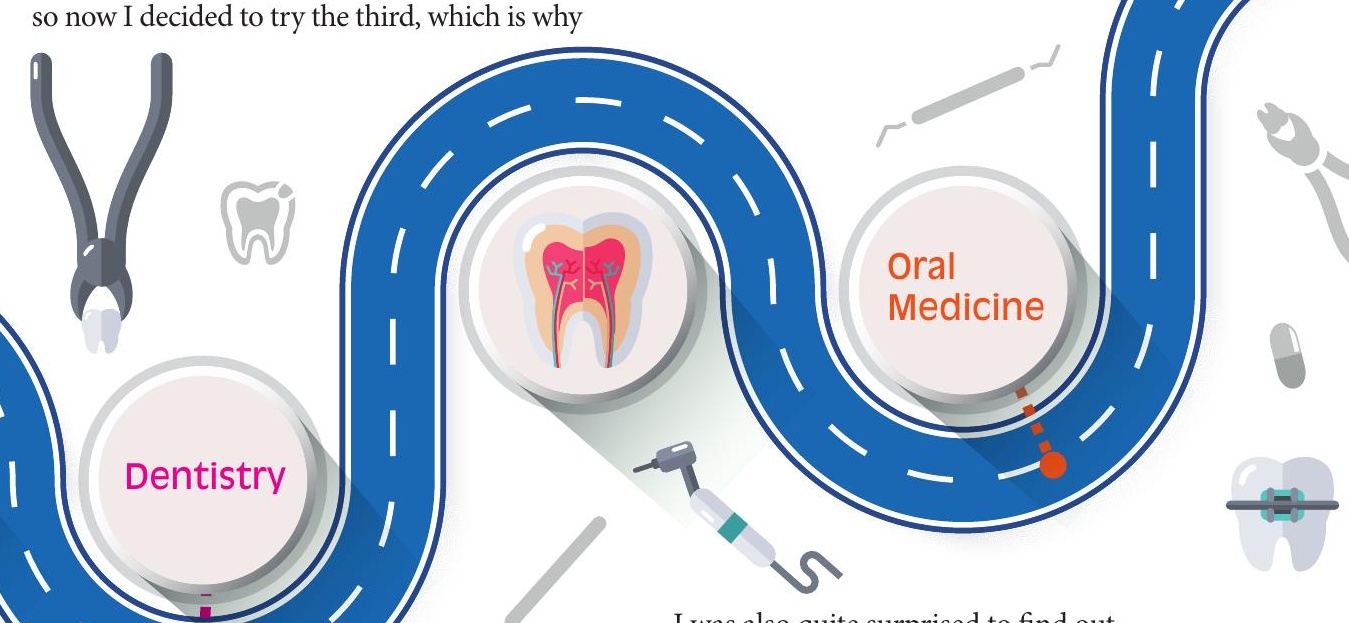

I was also quite surprised to find out that not all dental trainees have membership of a Royal College. That's probably because of my medical training and background - you cannot gain entry to training in any medical speciality without this. In medicine the Royal Colleges seem to play a much larger part in the setting the standards and structuring postgraduate training than they do in dentistry where the specialist societies seem to play a larger role. Though when I look at the Speciality Training Curricula for some of the medical specialities, I really think that there is scope for the Royal Colleges to play a larger part in postgraduate dental training. At the end of the day, training in dentistry always seems to follow medicine!

I have no career regrets so far. Despite numerous career setbacks I feel I have been lucky and I have been able to follow my interests. There have been times when my career has had to take a back seat to other priorities but I have also travelled the world and met some great people along the way. I seem to come across a lot of people at the Royal College who have the view that the best people are not the ones who have an easy or straightforward path to success, but those who struggle and despite obstacles persevere to achieve. 